\title{
Short-Term Effects of a Deterioration of General Health on the Oral Health of Nursing-Home Residents
}

\author{
Anna-Luisa Klotz $\mathbb{D}^{\prime}$ \\ Melania Zajac' \\ Judith Ehret' \\ Samuel Kilian ${ }^{2}$ \\ Peter Rammelsberg' \\ Andreas Zenthöfer ${ }^{\prime}$ \\ 'University of Heidelberg, Dental School, \\ Department of Prosthetic Dentistry, \\ Heidelberg 69I20, Germany; ${ }^{2}$ University \\ of Heidelberg, Institute of Medical \\ Biometry and Informatics, Heidelberg \\ 69120 , Germany
}

This article was published in the following Dove Press journal: Clinical Interventions in Aging

\begin{abstract}
Purpose: The purpose of this study was to identify how changes to general health might
\end{abstract} affect the oral health of nursing-home residents over a six-month period.

Materials and Methods: The study was conducted in nine nursing homes in Germany. Sociodemographic and general data were collected at baseline and after six months. Complete baseline and follow-up data were available for 114 participants. The Clinical Dementia Rating (CDR) and the Global Deterioration Scale (GDS) were used to identify the presence and, if applicable, severity of dementia among participants. The Apraxia Screen of TULIA (AST) was used to identify motor impairment. A comprehensive dental examination of each participant was also performed. The examination included the documentation of dental and denture status and the number of decayed, missing and filled teeth (DMF-T). In addition, dental and denture hygiene were quantitatively assessed using the Plaque Index (PI) and the Denture Hygiene Index (DHI), respectively. Global dental treatment needs were evaluated by use of the Oral Health Assessment Tool (OHAT). Univariate and multivariate linear regression models were compiled to analyse possible factors affecting the dependent target variables.

Results: During the study period, denture hygiene among the study population worsened by an average of $15 \%$. The regression models detected that a more advanced age (in years) was associated with a less pronounced deterioration of denture hygiene as measured by use of the DHI $(-0.806$ per additional year of age; $p=0.030)$. Furthermore, an increase in the level of care needed (coefficient $(\mathrm{C}):-1.948 ; p=0.002$ ) and a more poorly graded assessment of general health compared with the value at baseline $(\mathrm{C}: 1.054 ; p=0.026)$ were both associated with a deterioration of oral health as evaluated by use of the OHAT. In addition to these results, an increase in care needs was also associated with a deterioration of dental health as evaluated by use of the DMF-T (C: 0.966; $p=0.013$ ).

Conclusion: In the short term, a deterioration of general health, including an increase in care needs, seems to be the predominant factor associated with a more pronounced deterioration of oral health among nursing-home residents.

Keywords: oral health, oral hygiene, caries, older people, nursing home

\section{Introduction}

Ageing is a substantial risk factor for the development of general health problems, including cognitive and motor impairments. ${ }^{1}$ Many older people have specialised care needs and are therefore dependent on nursing homes. Because many Western countries are experiencing the long-term trend of an ageing population, the general and oral health of older people is becoming an increasingly important research subject.
Correspondence: Anna-Luis
University of Heidelberg, Im

Neuenheimer Feld 400, Heidelberg

69120 , Germany

Tel $+49622 I 566082$

Fax $+49622156537 \mid$

Email anna-luisa.klotz@med.uni-

heidelberg.de 
Several studies have found that the oral health of selfreliant older people has improved. ${ }^{2,3}$ The oral health of nursing-home residents, in contrast, is still unsatisfactory; this is because the prevalence of oral-health problems such as untreated caries, periodontal diseases and edentulism is still high. ${ }^{4-7}$ The main cause of such oral diseases is inadequate daily oral hygiene, which is a substantial problem for people in long-term care. ${ }^{8-10}$ These oral-health problems can be aggravated by cognitive and motor impairments and a decline in general health. Many older people with dementia refuse the administration of daily oral hygiene, and their caregivers might therefore experience a conflict between respecting the patient's autonomy and providing good daily care. Furthermore, as a patient's general health worsens, their dental hygiene and health are often neglected. ${ }^{6,11}$ However, several studies have found associations between general health and oral health. In this context, a deterioration of oral health seems to affect a person's general health by aggravating cardiovascular diseases and infectious respiratory diseases. ${ }^{12-15}$ The interconnection of oral health and cognition in several ways has also been described. ${ }^{1,16}$ As far as the authors of this study are aware, however, few studies have used a longitudinal study design to examine how a deterioration of general health affects the oral health and hygiene of nursing-home residents. This information would, however, be of use for improving the oral health of nursing-home residents. The objective of this study was, therefore, to identify the effects of changes to general health - including the aggravation of cognitive and motor problems and an increase in care needs - on the deterioration of oral hygiene (using the PI and DHI) and oral health (using DMF-T and OHAT) among nursing-home residents.

\section{Methods}

\section{Study Setting}

This study was approved by the local review board of the University of Heidelberg (approval number S-420/2016). After approval, nine long-term care facilities in BadenWürttemberg and Hesse, two of the 16 federal states of Germany, were selected for the study. A dentist provided all residents and, if applicable, their legal representatives with written and oral information about the study. All residents and their legal representatives were then asked to participate in the study and to give written and oral consent. Because the study protocol required a follow-up examination after six months, all residents who planned to move nursing home during the study period were excluded from participating. There were no other exclusion criteria. The baseline examination of the study included 150 participants. To enable evaluation of the longitudinal research question, only participants with both a baseline and a sixmonth follow-up examination were analysed. Thus, 114 participants were available for the final analysis.

\section{General Health and Cognitive Status}

The following information was obtained from participants' medical and care files: age (in years), gender $(0=$ female, $1=$ male), number of diseases, regular medications, ongoing duration of nursing-home residence (in months) and level of care needed. The level of care needed was defined in accordance with the German care-insurance grading system, which categorises care needs into five grades. Participants with no care needs were classified as grade 0 . Those with minor and considerable care needs were classified as grades 1 and 2, respectively. Grade 3 included residents with high care needs, whereas grade 4 included those with very high care needs. Residents with very high care needs and additional, specialised nursing requirements were classified as grade 5 . The estimation of health condition of participants was also evaluated subjective by the dentist using a three-point scale $(\operatorname{good}=1$, reduced $=2$, poor $=3$ ). In this context the dentist included the perception of participants' constitution after assessment of all general health variables and cognitive status. Beside these variables personal hygiene, mobility, activity and self-sufficiency were also evaluated and included for final classification.

The presence and severity of dementia were evaluated by use of the Clinical Dementia Rating $\left(\mathrm{CDR} ;{ }^{17}\right)$ and the Global Deterioration Scale (GDS; ${ }^{18}$ ). The CDR is based on a five-point Likert scale and enables the characterisation of six domains of cognitive and functional performance: memory, orientation, judgement and problem solving, community affairs, home and hobbies, and personal care. Each domain was assessed by means of a structured interview with the senior and a second person (family member, care giver) ${ }^{17}$ Interviews were performed by two dentists trained at the Memory Clinic of the University of Heidelberg before the start of the study. The CDR scores were as follows: $0=$ no dementia; $0.5=$ very mild dementia; $1=$ mild dementia; $2=$ moderate dementia; 3 = severe dementia. A CDR score was recorded for each of the six domains, and the highest score from the six domains was used to calculate the final score. ${ }^{19}$ The 
GDS classifies cognitive status into seven grades. Grades 1-3 are defined as pre-dementia stages because grade 1 equates to no impairment and grade 2 and 3 to the personal perception of complaints. Grades 4-7 are defined as stages of dementia of increasing severity (mild dementia to severe dementia). The graded classification of dementia was based on an individual evaluation by the dentist after the interview and a personal conversation with the participant. $^{18}$ The dentists received training for both indices and were calibrated by a psychologist of the Department of Psychology of the University of Heidelberg before the start of the study.

The presence and severity of apraxia were assessed by use of the Apraxia Screen of TULIA (AST; ${ }^{20}$ ). The AST comprises 12 items that require participants to perform tasks in the pantomime and imitation domains (five items in the pantomime domain, seven items in the imitation domain). A dichotomous scoring system is used for each item $(1=$ pass and $0=$ fail $)$, thus the maximum score for the AST is 12. This maximum score is indicative of an absence of apraxia. ${ }^{20}$

\section{Oral and Denture Hygiene and Oral Health}

The dental examinations were performed by two dentists trained at the Department of Prosthodontics of the University of Heidelberg. Each participant underwent a comprehensive dental examination that included the assessment of their dental and prosthetic status. For analytical purposes, the type of prosthesis worn was categorised as follows: 1. Natural teeth or fixed dental prosthesis (FDP); 2. Removable dental prosthesis (RDP); 3. Complete denture (CD); 4. Edentulous without dental prosthesis (ENP). For the variable "total denture status", each participant was classified according to the weaker restored jaw. $^{21}$

The Plaque Index (PI) and Denture Hygiene Index (DHI) were used to evaluate oral and denture hygiene, respectively. The PI evaluates quantitative plaque accumulation on natural teeth and is graded on a four-point scale $(0=$ no plaque to $3=$ substantial plaque accumulation $)$ before the index value is then divided by the number of surfaces assessed. ${ }^{22}$ The DHI was used to quantify denture hygiene (range: $0-100 \%$ ). Higher DHI values are indicative of poorer denture hygiene. ${ }^{23}$

Furthermore, decayed (D), missing (M) and filled (F) teeth were recorded by use of the DMF-T index to evaluate dental health status. In this study, third molars were excluded from calculations. Scores for D, M and F could therefore range from 0 to 28 in each case. ${ }^{24}$ In addition to the DMF-T, the oral health and dental- or denture-related treatment needs were also evaluated by use of the German version of the Oral Health Assessment Tool (OHAT; ${ }^{25}$ ). The OHAT has been described as a reliable and effective assessment tool for evaluating the oral health of nursing-home residents with and without dementia and contains eight items relating to objective aspects of oral health: lips, tongue, gums and tissues, saliva, natural teeth, dentures, oral cleanliness and dental pain. For each category, the examiner scores the condition on a three-point scale: $0=$ healthy; $1=$ changes; $2=$ unhealthy. The total OHAT score can therefore range from a minimum of 0 points to a maximum of 16 points. ${ }^{26}$

\section{Statistical Evaluation}

Mean values, standard deviations, counts and frequencies were used to descriptively present the results of the baseline and follow-up examinations. Results were given as means $( \pm \mathrm{SD})$ or counts (frequency \%). Furthermore a drop out analysis was conducted for baseline characteristics of the participants.

A univariate linear regression model was used to assess the longitudinal association of participants' characteristics and changes to general health with changes to the dependent variables (PI, DHI, DMF-T, OHAT). This was performed for each covariate/factor and each dependent variable. A change to continuous variables (number of diseases, number of medications, PI, DHI, DMF-T, OHAT) was defined as the difference between the baseline and follow-up assessments. A change to ordinally scaled variables (care level, general health, CDR, GDR, AST, presence of own teeth) was defined dichotomously as a deterioration of the score $(0=$ equal/better; $1=$ worse $)$. Furthermore, multivariate linear regression was conducted after a stepwise selection of variables using the $p$-value to capture the factors most strongly associated with a change in the dependent dental variables. The stepwise variable selection algorithm firstly excluded all variables which had a p-value greater than 0.5 in the univariate linear regressions and performed secondly a backward step, where the variable with the highest p-value was excluded. Thirdly, it checked for every not included variable whether the p-value, if re-included in the model, was below the threshold of 0.05 . If so, the excluded variable with the smallest p-value had been re-included (forward step). The 
backward and forward steps were reiterated until all included variables were significant or only one variable was left. Variables with a $p$-value of $\leq 0.05$ were included in the final model.

Statistical analysis was performed by use of the software R, version 3.4.2 (R Core Team; Auckland, New Zealand). $P$-values of less than 0.05 were regarded as significant.

\section{Results}

\section{Study Population}

The mean age of the participants at baseline was $82.0( \pm 9.5)$ years, and $77.2 \%$ of the participants were female. Estimation of health condition at baseline was evaluated as good for $62(54.4 \%)$ participants, reduced for $39(34.2 \%)$ and poor for $13(11.4 \%)$ participants. After six months, the estimation of health condition of the participants was worse than it was at baseline (good: $48(42.1 \%)$, reduced: 50 (43.9\%), poor: $16(14.0 \%))$. The care levels at baseline among all participants were: care level 0 , three participants (2.6\%); care level 1 , four participants $(3.5 \%)$; care level 2 , 38 participants $(33.3 \%)$; care level 3, 35 participants (30.7\%); care level 4, 28 participants (24.6\%); care level 5,6 participants $(5.3 \%)$. The mean number of diseases at baseline was $5.9( \pm 3.5)$, and the mean number of medications taken was $8.7( \pm 3.7)$. Most participants $(86.8 \%)$ had at least mild dementia $(C D R>0)$ at baseline. The mean value of the AST was $11.0( \pm 2.8)$. The mean BMI at baseline was 27.7 ( \pm 6.6$)$. The characteristics of the study drop-outs were comparable to those of the participants who completed the study. Detailed results are given in Table 1.

\section{Oral and Denture Hygiene and Oral Health}

The mean number of remaining teeth among the participants was $9.2 \pm 9.6$ at baseline and $8.9 \pm 9.3$ after six months. Thirty-one participants $(27.2 \%)$ wore a fixed dental prosthesis (FDP) or had natural remaining teeth ; $19(16.7 \%)$ wore a removable dental prosthesis (RDP); and $50(43.9 \%)$ wore a complete denture (CD) in at least one jaw. Fourteen participants $(12.3 \%)$ were edentulous and wore no dental prosthesis (ENP). After six months, the number of participants with an FDP or natural teeth had not changed. However, the number of participants with an RDP $(17 / 14.9 \%)$ or CD (22/19.3\%) had decreased, whereas the proportion of edentulous participants had increased to $22(19.3 \%)$. The mean PI, DHI and DMF-T at baseline were $2.1 \pm 0.9,54.5 \pm 27.5$ and $25.4 \pm 4.0$, respectively. After six months, the PI, DHI and DMF-T scores increased signiticanlty to $2.4 \pm 0.8$, $69.9 \pm 27.5$ and $26.0 \pm 3.6$, respectively. On consideration of decayed teeth at baseline, 18 participates $(94 / 7 \%)$ with RDP had decayed teeth. The mean OHAT of the participants was $5.8 \pm 2.4$ at baseline and $6.5 \pm 2.4$ at the follow-up examination. Detailed results are shown in Table 1.

\section{Univariate Regression Analysis}

Univariate regression analysis detected that a more advanced age was associated with less pronounced deterioration of the DHI score (C: $-0.806 ; p=0.030)$. Furthermore, an increase in care needs was associated with a greater difference in OHAT score and, therefore, with a greater deterioration of the OHAT score $(\mathrm{C}:-2.098 ; p=0.001)$. On average, the difference in the OHAT score of participants whose care needs had increased was 2.098 higher than that of the other participants. With regard to general health, the univariate regression model detected that a deterioration of the estimation of health condition of the participants was associated with a higher difference in OHAT score and, therefore, a worse OHAT score (C: $1.211 ; p=0.013)$. In this context, the increase in the OHAT score of participants whose estimated health condition had deteriorated was on average 1.211 times higher than that of the other participants. Furthermore, an increase in care needs was associated with a greater deterioration of the DMF-T score $(\mathrm{C}: 0.966 ; p=0.013)$. In this context, the increase in the DMF-T score of participants with increased care needs was on average 0.966 times higher than that of participants with no increase in care needs.

None of the other variables analysed were substantially associated with the endpoints. Detailed results of the univariate regression analysis are given in Table 2 .

\section{Multivariate Linear Regression Analysis}

Almost all final models of the variable selection were univariate or empty and thus yielded no further insights. Only the final model for the change to OHAT scores contained the two covariates of increased care needs $(\mathrm{C}:-1.948, p=0.002)$ and deterioration of the estimation of health condition ( $\mathrm{C}: 1.054, p=0.026)$. This suggests that both variables had a simultaneous effect on the change to OHAT scores. Detailed results are shown in Table 3. 
Table I Participant Characteristics at Baseline and After Six Months and Drop-Outs. Data are Presented as Means (SD) or Counts (Frequency)

\begin{tabular}{|c|c|c|c|}
\hline & Drop-Outs $(n=36)$ & Participants Analysed at Baseline $(n=114)$ & $\begin{array}{l}\text { Six-Month Follow-Up of the } \\
\text { Analysed Participants }\end{array}$ \\
\hline Age & $82.4(10.8)$ & $82.0(9.5)$ & $82.5(9.5)$ \\
\hline \multicolumn{4}{|l|}{ Gender } \\
\hline Female & 25 (69.4\%) & $88(77.2 \%)$ & $88(77.2 \%)$ \\
\hline Male & II (30.6\%) & $26(22.8 \%)$ & $26(22.8 \%)$ \\
\hline Number of teeth & $12.8(9.9)$ & $9.2(9.6)$ & $8.9(9.3)$ \\
\hline \multicolumn{4}{|l|}{ Level of care } \\
\hline 0 & I (2.8\%) & $3(2.6 \%)$ & $2(1.8 \%)$ \\
\hline 1 & $2(5.6 \%)$ & $4(3.5 \%)$ & $3(2.6 \%)$ \\
\hline 2 & 7 (19.4\%) & $38(33.3 \%)$ & $36(31.6 \%)$ \\
\hline 3 & $11(30.6 \%)$ & $35(30.7 \%)$ & $37(32.5 \%)$ \\
\hline 4 & $14(38.9 \%)$ & $28(24.6 \%)$ & $27(23.7 \%)$ \\
\hline 5 & $\mathrm{I}(2.8 \%)$ & $6(5.3 \%)$ & $9(7.9 \%)$ \\
\hline Number of diseases & $6(3.4)$ & $5.9(3.5)$ & $6.5(3.4)$ \\
\hline Number of medications & $7.2(3.4)$ & $8.7(3.7)$ & $8.8(4.2)$ \\
\hline \multicolumn{4}{|l|}{ Total denture status } \\
\hline FDP/natural teeth & $14(38.9 \%)$ & $31(27.1 \%)$ & $31(27.1 \%)$ \\
\hline RDP & $8(22.2 \%)$ & $19(16.7 \%)$ & 17 (I4.9\%) \\
\hline$C D$ & $9(25.0 \%)$ & 50 (43.9\%) & $44(38.6 \%)$ \\
\hline ENP & $5(13.9 \%)$ & $14(12.3 \%)$ & $22(19.3 \%)$ \\
\hline PI $(n=77)$ & $2.3(0.8)$ & $2.1(0.9)$ & $2.4(0.8)$ \\
\hline DHI total $(n=74)$ & $53.9(32.3)$ & $54.5(27.5)$ & $69.9(27.5)$ \\
\hline Decayed teeth & $1.7(1.8)$ & $1.8(3.3)$ & $2.9(4.0)$ \\
\hline Missing teeth & $15.3(9.7)$ & $19.0(9.3)$ & $19.2(9.6)$ \\
\hline Filled teeth & $6.9(6.6)$ & $4.7(5.9)$ & $3.8(5.3)$ \\
\hline DMF-T & $23.9(4.8)$ & $25.4(4.0)$ & $26.0(3.6)$ \\
\hline \multicolumn{4}{|l|}{ Estimation of health condition } \\
\hline Good & 19 (52.8\%) & $62(54.4 \%)$ & $48(42.1 \%)$ \\
\hline Reduced & $12(33.3 \%)$ & 39 (34.2\%) & $50(43.9 \%)$ \\
\hline Poor & $5(13.9 \%)$ & $13(2.4 \%)$ & $16(14.0 \%)$ \\
\hline ОНАТ & $5.4(2.3)$ & $5.8(2.4)$ & $6.5(2.4)$ \\
\hline \multicolumn{4}{|l|}{ GDR } \\
\hline 0.5 & $0(0.0 \%)$ & I (0.9\%) & $9(7.9 \%)$ \\
\hline 1 & $5(13.9 \%)$ & $16(14.0 \%)$ & $34(29.8 \%)$ \\
\hline 2 & II (30.6\%) & 31 (27.2\%) & 19 (16.6\%) \\
\hline 3 & $5(13.9 \%)$ & 20 (17.5\%) & 15 (I3.2\%) \\
\hline 4 & 5 (13.9\%) & 14 (I2.3\%) & 12 (10.5\%) \\
\hline 5 & I (2.8\%) & 12 (10.5\%) & 14 (12.3\%) \\
\hline 6 & 7 (19.4\%) & 12 (10.5\%) & II (9.6\%) \\
\hline 7 & $2(5.6 \%)$ & $8(7.0 \%)$ & 36 (31.6\%) \\
\hline \multicolumn{4}{|l|}{ CDR } \\
\hline 0 & 4 (11.1\%) & 15 (I3.2\%) & $8(7.0 \%)$ \\
\hline 0.5 & 12 (33.3\%) & 25 (21.9\%) & 25 (21.9\%) \\
\hline 1 & $6(16.7 \%)$ & 30 (26.3\%) & $28(24.6 \%)$ \\
\hline
\end{tabular}

(Continued) 
Table I (Continued).

\begin{tabular}{|l|l|l|l|}
\hline & Drop-Outs $(\mathbf{n}=\mathbf{3 6})$ & Participants Analysed at Baseline $(\mathbf{n}=1$ I 4) & $\begin{array}{l}\text { Six-Month Follow-Up of the } \\
\text { Analysed Participants }\end{array}$ \\
\hline 2 & $7(19.4 \%)$ & $22(19.3 \%)$ & $28(24.6 \%)$ \\
3 & $7(19.4 \%)$ & $22(19.3 \%)$ & $25(21.9 \%)$ \\
\hline AST $(\mathbf{n}=108)$ & $10.4(3.5)$ & $11.04(2.8)$ & $10.7(3.5)$ \\
\hline BMI & $26.6(5.5)$ & $27.7(6.6)$ & $27.8(6.8)$ \\
\hline
\end{tabular}

Abbreviations: FDP, fixed dental prosthesis; RDP, removable dental prosthesis; CD, complete denture; ENP, edentulous without dental prosthesis. PI, Plaque Index; DHI, Denture Hygiene Index; OHAT, Oral Health Impact Tool; GDR, Global Deterioration Scale; CDR, Clinical Dementia Rating; AST, Apraxia Screening Test.

Table 2 Univariate Regression Analysis with Dental Target Variables as Dependent Variables and Changes to General Health Over the Study Period as Independent Variables

\begin{tabular}{|c|c|c|c|c|}
\hline Variable & C & 95\% CI LB & $95 \% \mathrm{CI}$ UB & $P$-value \\
\hline \multicolumn{5}{|l|}{ Difference of PI $(n=77)$} \\
\hline Age & 0.003 & -0.018 & 0.025 & 0.758 \\
\hline Gender & -0.268 & -0.725 & 0.189 & 0.246 \\
\hline Deterioration of presence of own teeth * & -0.890 & -2.100 & 0.321 & 0.147 \\
\hline Deterioration of care level $*$ & 0.013 & -0.568 & 0.594 & 0.964 \\
\hline Difference of the number of diseases & 0.008 & -0.089 & 0.106 & 0.866 \\
\hline Difference of the number of medications & -0.052 & -0.136 & 0.033 & 0.227 \\
\hline Deterioration of the estimation of health condition * & 0.236 & -0.206 & 0.678 & 0.292 \\
\hline Deterioration of GDR* & 0.267 & -0.155 & 0.689 & 0.212 \\
\hline Deterioration of CDR* & 0.050 & -0.377 & 0.476 & 0.816 \\
\hline Deterioration of AST $(n=108) *$ & 0.019 & -0.686 & 0.724 & 0.957 \\
\hline \multicolumn{5}{|l|}{ Difference of DHI $(n=74)$} \\
\hline Age & -0.806 & -1.534 & -0.078 & 0.030 \\
\hline Gender & 3.707 & $-|5.86|$ & 22.876 & 0.701 \\
\hline Deterioration of presence of own teeth* & -0.417 & -45.446 & 44.612 & 0.985 \\
\hline Deterioration of care level * & -5.714 & -37.978 & 26.550 & 0.725 \\
\hline Difference of the number of diseases & -1.272 & -5.106 & 2.561 & 0.510 \\
\hline Difference of the number of medications & -1.127 & -4.385 & 2.130 & 0.493 \\
\hline Deterioration of the estimation of health condition * & $13.97 \mid$ & -2.419 & 30.361 & 0.094 \\
\hline Deterioration of GDR* & 3.717 & -6.744 & 26.962 & 0.236 \\
\hline Deterioration of CDR* & 10.109 & -6.744 & 26.962 & 0.236 \\
\hline Deterioration of AST $(n=108) *$ & 8.147 & -15.840 & 32.135 & 0.500 \\
\hline \multicolumn{5}{|l|}{ Difference of OHAT $(n=114)$} \\
\hline Age & 0.000 & -0.042 & 0.043 & 0.984 \\
\hline Gender & -0.622 & -1.569 & 0.326 & 0.196 \\
\hline Deterioration of presence of own teeth* & -0.214 & -3.264 & 2.835 & 0.890 \\
\hline Deterioration of care level* & -2.098 & -3.342 & -0.854 & 0.001 \\
\hline Difference of the number of diseases & -0.076 & -0.268 & 0.116 & 0.434 \\
\hline Difference of the number of medications & 0.032 & -0.152 & 0.215 & 0.734 \\
\hline Deterioration of the estimation of health condition * & 1.211 & 0.255 & 2.167 & 0.013 \\
\hline Deterioration of GDR* & 0.005 & -0.863 & 0.874 & 0.990 \\
\hline Deterioration of CDR* & 0.619 & -0.293 & 1.532 & 0.181 \\
\hline Deterioration of AST $(n=108) *$ & -1.087 & -2.458 & 0.284 & 0.119 \\
\hline \multicolumn{5}{|l|}{ Difference of DMF-T $(n=114)$} \\
\hline Age & -0.015 & -0.040 & 0.010 & 0.227 \\
\hline Gender & 0.081 & -0.486 & 0.648 & 0.777 \\
\hline Deterioration of presence of own teeth* & -0.562 & -2.372 & 1.247 & 0.539 \\
\hline Deterioration of care level $*$ & 0.966 & 0.212 & 1.720 & 0.013 \\
\hline
\end{tabular}

(Continued) 
Table 2 (Continued).

\begin{tabular}{|c|c|c|c|c|}
\hline Variable & C & $95 \%$ CI LB & $95 \% \mathrm{CI}$ UB & $P$-value \\
\hline Difference of the number of diseases & -0.037 & -0.152 & 0.077 & 0.519 \\
\hline Difference of the number of medications & -0.062 & -0.171 & 0.046 & 0.257 \\
\hline Deterioration of the estimation of health condition * & 0.250 & -0.332 & 0.832 & 0.396 \\
\hline Deterioration of GDR* & 0.192 & -0.323 & 0.707 & 0.461 \\
\hline Deterioration of $\mathrm{CDR}^{*}$ & -0.232 & -0.777 & 0.312 & 0.400 \\
\hline Deterioration of AST $(\mathrm{n}=108) *$ & 0.171 & -0.656 & 0.997 & 0.683 \\
\hline \multicolumn{5}{|l|}{ Difference of decayed teeth $(n=114)$} \\
\hline Age & -0.014 & -0.063 & 0.036 & 0.592 \\
\hline Gender & -0.277 & -1.398 & 0.843 & 0.625 \\
\hline Deterioration of presence of own teeth* & -1.705 & 5.276 & 1.865 & 0.346 \\
\hline Deterioration of care level $*$ & 1.760 & 0.262 & 3.258 & 0.022 \\
\hline Difference of the number of diseases & -0.104 & -0.330 & 0.122 & 0.363 \\
\hline Difference of the number of medications & -0.056 & -0.272 & 0.160 & 0.606 \\
\hline Deterioration of the estimation of health condition * & -0.433 & -1.585 & 0.718 & 0.458 \\
\hline Deterioration of GDR* & 0.365 & -0.653 & 1.383 & 0.479 \\
\hline Deterioration of CDR* & -0.328 & -1.407 & 0.751 & 0.548 \\
\hline Deterioration of AST $(\mathrm{n}=108) *$ & 0.067 & -1.566 & 1.699 & 0.936 \\
\hline \multicolumn{5}{|l|}{ Difference of missing teeth $(n=114)$} \\
\hline Age & -0.007 & -0.024 & 0.010 & 0.390 \\
\hline Gender & -0.003 & -0.383 & 0.376 & 0.985 \\
\hline Deterioration of presence of own teeth* & 0.741 & -0.465 & 1.947 & 0.226 \\
\hline Deterioration of care level $*$ & -0.025 & -0.554 & 0.495 & 0.926 \\
\hline Difference of the number of diseases & 0.022 & -0.055 & 0.098 & 0.579 \\
\hline Difference of the number of medications & 0.013 & -0.060 & 0.086 & 0.719 \\
\hline Deterioration of the estimation of health condition * & -0.133 & -0.523 & 0.257 & 0.500 \\
\hline Deterioration of GDR* & -0.021 & -0.367 & 0.324 & 0.903 \\
\hline Deterioration of CDR* & -0.041 & -0.407 & 0.325 & 0.825 \\
\hline Deterioration of AST $(n=108) *$ & 0.197 & -0.356 & 0.749 & 0.481 \\
\hline \multicolumn{5}{|l|}{ Difference of filled teeth $(n=114)$} \\
\hline Age & 0.008 & -0.028 & 0.045 & 0.647 \\
\hline Gender & 0.328 & -0.488 & 1.143 & 0.428 \\
\hline Deterioration of presence of own teeth* & 0.375 & -2.238 & 2.988 & 0.777 \\
\hline Deterioration of care level $*$ & -0.799 & -1.907 & 0.309 & 0.156 \\
\hline Difference of the number of diseases & 0.042 & -0.123 & 0.207 & 0.002 \\
\hline Difference of the number of medications & -0.022 & -0.180 & 0.136 & 0.783 \\
\hline Deterioration of the estimation of health condition * & 0.889 & 0.064 & 1.714 & 0.035 \\
\hline Deterioration of GDR* & -0.066 & -0.810 & 0.678 & 0.860 \\
\hline Deterioration of CDR* & 0.147 & -0.640 & 0.935 & 0.712 \\
\hline Deterioration of AST $(n=108) *$ & 0.079 & -1.107 & 1.264 & 0.896 \\
\hline
\end{tabular}

Notes: Significant $p$-values are marked in bold.*Participant characteristics are reported in a binary manner.

Abbreviations: C, regression coefficient; LB, lower boundary; UB, upper boundary. PI, Plaque Index; DHI, Denture Hygiene Index; OHAT, Oral Health Impact Tool; GDR, Global Deterioration Scale; CDR, Clinical Dementia Rating; AST, Apraxia Screening Test.

Table 3 Multivariate Linear Regression Model After Stepwise Selection of Variables Using the $p$-Value with Different Dependent Variables

\begin{tabular}{|c|c|c|c|c|}
\hline Variable & Coefficient & $\begin{array}{l}95 \% \mathrm{Cl} \\
\text { LB }\end{array}$ & $\begin{array}{l}95 \% \mathrm{Cl} \\
\text { UB }\end{array}$ & $p$-value \\
\hline \multicolumn{5}{|l|}{ Difference of OHAT $(n=|| 4)$} \\
\hline Intercept & 0.694 & 0.246 & 1.142 & 0.003 \\
\hline Deterioration of the estimation of health condition $*$ & 1.054 & 0.129 & 1.980 & 0.026 \\
\hline Deterioration of care level $*$ & -1.948 & -3.177 & -0.719 & 0.002 \\
\hline
\end{tabular}

Notes: Significant $p$-values are marked in bold. *Participant characteristics are reported in a binary manner.

Abbreviation: OHAT, Oral Health Assessment Tool. 


\section{Discussion}

The results of this study suggest that a decline in general health, including an increase in care needs, is the predominant factor associated with a decline in oral health among nursing-home residents, at least in the short term.

With regard to participant characteristics, it is notable that the general and dental health of participants were comparable to those found in recent studies of nursinghome residents. ${ }^{4-7,26}$ However, it was surprising that both the general and dental health of the participants substantially worsened during the quite short observation period of six months. On closer inspection of the dental variables, it is striking that the denture status did not change during the study period for any of the participants with an FDP or natural teeth. In contrast, changes to denture status were observed among participants with an RDP or CD. In these two groups, eight participants lost their teeth and their dental prosthesis and therefore had no prosthetic restoration at all. In addition to these changes, denture hygiene also worsened during the observation period, as did dental and oral health as evaluated by use of DMF-T and OHAT. In many cases, the general health of many participants, including the level of care needed, also deteriorated. In contrast, the cognitive status of participants as evaluated by use of the GDS and CDR did not change substantially during the observation period. This was to be expected because the observation period was too short to evaluate changes to cognitive status. The assessment of cognitive status therefore provides only a limited snapshot of the participants' cognitive status.

Closer inspection of the association between general health and dental hygiene, as evaluated by use of the PI, suggests that changes to the general health variables had very little effect on plaque accumulation on teeth. In crosssectional studies, however, greater plaque accumulation on the teeth of nursing-home residents has been associated with age, the presence of dementia and (male) gender. ${ }^{5}$ These differences might be explained by the fact that, in our study, cognitive status and PI did not change significantly during the observation period. To obtain significant results with regard to the relationship between cognitive status and PI, a longer study period might be required. Furthermore, it should be kept in mind that the PI only provides a snapshot of oral hygiene because the PI assessment was recorded at different times of day (sometimes in the morning after tooth brushing and sometimes after eating). With regard to denture hygiene, an association between a more advanced age and a less severe decline in denture hygiene was observed. This result initially seems surprising because an advanced age is associated with a higher risk of poor general health, motor impairment and cognitive deficits. This in turn results in a loss of ability to perform daily oral hygiene. ${ }^{27}$ However, it is possible that the caregivers in this study setting took better care of older participant's oral health care. On the other hand it is also possible that the denture hygiene of older participants was wore at baseline and could therefore not deteriorate that much compared to younger participants with better denture hygiene at baseline.

Nevertheless, the analysis of dental and oral health as evaluated by use of the DMF-T and OHAT yielded convincing results. Important influencing factors could thus be determined for both parameters. Closer inspection of the DMF-T and OHAT revealed that a deterioration of the estimation of health condition and an increase in care needs were most strongly associated with a decline in the dental and oral-health condition of participants. As the number of decayed teeth increased during the study period, it is also possible that saliva secretion, which is often a side effect of medications, reduced. This is an important influencing factor for dental caries. The fact that the OHAT also got worse during the study period underlines this theory as the OHAT contains the item saliva.

Considering these results, it is possible that oral health becomes less important to nursing-home residents as their general health deteriorates. More oral-health support from caregivers in consequently needed as soon as this deterioration starts; however, similar to the attitude of the nursing-home residents, caregivers might devote less attention to oral-health care as a result of prioritising their residents' general health. In addition, many nursinghome residents refuse the assistance of caregivers. ${ }^{6,28}$ This shift of priorities leads to more dental problems, resulting in the loss of remaining teeth and a decline in prosthetic status. This, in turn, results in a deterioration of oral health. In this context, it should be kept in mind that a decline in oral health not only affects chewing function, it also affects the oral-health-related quality of life and general health of nursing-home residents by aggravating systemic diseases. This consequently results in a greater risk of mortality. ${ }^{28,29}$ Interestingly, a decline in cognitive status did not significantly affect dental and oral health in our study. This might be due to the quite short observation period and minor changes in cognitive status. 
Based on the results of this study, it seems strongly advisable to periodically evaluate the dental and oral health of nursing-home residents. Moreover, specific attention should be paid to dental and oral health as soon a deterioration of general health is detected. Caregivers could use the OHAT to perform regular check-ups, for example, in addition to dentists conducting home visits.

\section{Strengths and Weaknesses of the Study}

All eligible participants who wanted to participate and gave written and oral consent were included in this study, irrespective of their cognitive status and care needs. This was a strength of this study because it meant that a wide range of nursing-home residents was studied. However, it is possible that those participants who chose to participate were more interested in their oral health than non-responders. It is also possible that some participants suspected they had acute dental problems and participated for this reason. It should also be kept in mind that only participants who underwent two examinations were included in the study. Twenty-four per cent of nursing-home residents could therefore not be included. This might have resulted in bias; however, the drop-out analysis determined that the participant characteristics of both groups were comparable at baseline. It should also be noted that the dental and psychological examinations performed in this study were timeconsuming; for this reason, the psychological examinations were performed first to reduce bias. All other examinations were objective and therefore not dependent on the participation of the nursing-home resident. It should be noted that the number of diseases and the number of medications can only be considered as indicators of general health condition of participants, as seriousness of diseases is different and not considered in this study. It also should be considered that some participants received the maximum score in one of the estimated variables at baseline and could therefore not worsen in this variable in the observed period which might lead to a bias. One more weakness is that saliva flow rates were not assessed in this study.

\section{Ethical Approval}

All procedures performed in this study involving human participants were in accordance with the ethical standards of the institutional and/or national committee and with the 1964 Declaration of Helsinki and its later amendments or comparable ethical standards.

\section{Acknowledgments}

We are grateful to all the participants in this study for their patience and cooperation. We would also like to thank
Hazel Davies, copy editor, for the English-language revision. Furthermore, we would like to thank Christopher Büsch for statistical support. ALK was supported by the GEROK program of the University of Heidelberg.

\section{Disclosure}

The authors report no conflicts of interest regarding this manuscript.

\section{References}

1. Noble JM, Scarmeas N, Papapanou PN. Poor oral health as a chronic, potentially modifiable dementia risk factor: review of the literature. Curr Neurol Neurosci Rep. 2013;13(10):384.

2. Jordan AR, Micheelis W. Fünfte Deutsche Mundgesundheitsstudie $(D M S V)$. Köln: Institut der Deutschen Zahnärzte (IDZ Materialreihe Band 35), Deutscher Ärzte-Verlag; 2016.

3. Petersen PE, Ogawa H. Promoting oral health and quality of life of older people - the need for public health action. Oral Health Prev Dent. 2018;16(2):113-124.

4. Janssens B, Vanobbergen J, Petrovic M, Jacquet W, Schols JM, De Visschere L. The impact of a preventive and curative oral healthcare program on the prevalence and incidence of oral health problems in nursing home residents. PLoS ONE. 2018;13(6):e0198910. doi:10.1371/ journal.pone. 0198910

5. Thomson WM, Smith MB, Ferguson CA, Kerse NM, Peri K, Gribben B. Oral status, cognitive function and dependency among New Zealand nursing home residents. Gerodontology. 2018;35:185-191. doi:10.1111/ger.12337

6. Yoon MN, Ickert C, Slaughter SE, Lengyel C, Carrier N, Keller H. Oral health status of long-term care residents in Canada: results of a national cross-sectional study. Gerodontology. 2018;35(4):359-364. doi:10.1111/ger.2018.35.issue-4

7. Pham TAV, Nguyen TQ. Dental and periodontal problems of elderly people in Vietnamese nursing homes. Gerodontology. 2018;35:192-199. doi:10.1111/ger.12338

8. Chauncey HH, Glass RL, Alman JE. Dental caries. Principal cause of tooth extraction in a sample of US male adults. Caries Res. 1989;23 (3):200-205.

9. Bailit HL, Braun R, Maryniuk GA, Camp P. Is periodontal disease the primary cause of tooth extraction in adults? $J$ Am Dental Assoc. 1987;114(1):40-45.

10. Stephens RG, Kogon SL, Jarvis AM. A study of the reasons for tooth extraction in a Canadian population sample. J Can Dent Assoc. 1991;57(6):501-504.

11. Ástvaldsdóttir Á, Boström AM, Davidson T, et al. Oral health and dental care of older persons-A systematic map of systematic reviews. Gerodontology. 2018;35(4):290-304. doi:10.1111/ger.12368

12. Adachi M, Ishihara K, Abe S, Okuda K. Professional oral health care by dental hygienists reduced respiratory infections in elderly persons requiring nursing care. Int J Dent Hyg. 2007;5:69-74. doi:10.1111/ idh.2007.5.issue-2

13. Awano S, Ansai T, Takata Y, et al. Oral health and mortality risk from pneumonia in the elderly. $J$ Dent Res. 2008;87:334-339. doi:10.1177/ 154405910808700418

14. Juthani-Mehta M, De Rekeneire N, Allore H, Chen S, O'Leary JR, Bauer DC. Modifiable risk factors for pneumonia requiring hospitalization of community-dwelling older adults: the health, aging, and Body Composition Study. J Am Geriatr Soc. 2013;61:1111-1118. doi:10.1111/jgs.2013.61.issue-7

15. Scannapieco FA, Rethman MP. The relationship between periodontal diseases and respiratory diseases. Dent Today. 2003;22:79-83. 
16. Delwel S, Binnekade TT, Perez RS, Hertogh CM, Scherder EJ, Lobbezoo F. Oral health and orofacial pain in older people with dementia: a systematic review with focus on dental hard tissues. Clin Oral Investig. 2017;21(1):17-32. doi:10.1007/s00784-0161934-9

17. Morris JC. The Clinical Dementia Rating (CDR): current version and scoring rules. Neurology. 1993;43(11):2412-2414. doi:10.1212/ WNL.43.11.2412-a

18. Reisberg B, Ferris SH, de Leon MJ, Crook T. The Global Deterioration Scale for assessment of primary degenerative dementia. Am J Psychiatry. 1982;139(9):1136-1139.

19. Galvin JE, Meuser TM, Coats MA, Bakal DA, Morris JC. The "portable" CDR: translating the clinical dementia rating interview into a PDA format. Alzheimer Dis Assoc Disord. 2009;23(1):44-49. doi:10.1097/WAD.0b013e31817634a0

20. Vanbellingen T, Kersten B, Van de Winckel A, et al. A new bedside test of gestures in stroke: the apraxia screen of TULIA (AST). J Neurol Neurosurg Psychiatry. 2011;82(4):389-392. doi:10.1136/ jnnp.2010.213371

21. Hassel AJ, Koke U, Schmitter M, Rammelsberg P. Factors associated with oral health-related quality of life in institutionalized elderly. Acta Odontol Scand. 2016;64:9-15. doi:10.1080/ 00016350500326211
22. Silness J, Loe H. Periodontal disease in pregnancy. II. Correlation between oral hygiene and periodontal condition. Acta Odontol Scand. 1964;22:121-135.

23. Wefers AP. The "Denture Hygiene Index". Dental Forum. 1991;1:3-15.

24. World Health Organization. Oral health surveys: basic methods. Geneva, Switzerland: World Health Organization; 1987.

25. Klotz AL, Zajac M, Ehret J, Hassel AJ, Rammelsberg P, Zenthöfer A. Development of a German version of the Oral Health Assessment Tool. Aging Clin Exp Res. 2019. doi:10.1007/s40520-019-01158-x

26. Chalmers JM, King PL, Spencer AJ, Wright FA, Carter KD. The oral health assessment tool - Validity and reliability. Aust Dent J. 2005;50:191-199. doi:10.1111/j.1834-7819.2005.tb00360.x

27. Osterberg T, Mellström D, Sundh V. Dental health and functional ageing. A study of 70-year-old people. Community Dent Oral Epidemiol. 1990;18(6):313-318. doi:10.1111/com.1990.18.issue-6

28. Ajwani S, Mattila KJ, Närhi TO, Tilvis RS, Ainamo A. Oral health status, C-reactive protein and mortality-A 10 year follow-up study. Gerodontology. 2003;20(1):32-40. doi:10.1111/ger.2003.20.issue-1

29. Klotz AL, Hassel AJ, Schröder J, Rammelsberg P, Zenthöfer A. Is compromised oral health associated with a greater risk of mortality among nursing home residents? A controlled clinical study. Aging Clin Exp Res. 2018;30(6):581-588. doi:10.1007/s40520-017-0811-y
Clinical Interventions in Aging

\section{Publish your work in this journal}

Clinical Interventions in Aging is an international, peer-reviewed journal focusing on evidence-based reports on the value or lack thereof of treatments intended to prevent or delay the onset of maladaptive correlates of aging in human beings. This journal is indexed on PubMed Central, MedLine, CAS, Scopus and the Elsevier

\section{Dovepress}

Bibliographic databases. The manuscript management system is completely online and includes a very quick and fair peer-review system, which is all easy to use. Visit http://www.dovepress.com/ testimonials.php to read real quotes from published authors. 